\title{
Behavior of water molecules in ATPase pocket of myosin
}

\author{
Kazunori Yamanaka, ${ }^{* \dagger}$ Noriaki Okimoto, ${ }^{\ddagger}$ Masayuki Hata, ${ }^{*}$ Saburo Neya, ${ }^{*}$ and Tyuji Hoshino, ${ }^{\star \Uparrow}$ \\ *Graduate School of Pharmaceutical Sciences, Chiba University, Chiba 263-8522, Chiba, Japan ; \\ ${ }^{\dagger}$ Present Address: Banyu Tsukuba Research Institute, Tsukuba 300-2611, Ibaraki, Japan ; \\ ${ }^{\ddagger}$ Bioinformatics Group, GSC, RIKEN, 61-1 Ono-cho, Tsurumi, Yokohama 230-0046, Kanagawa, Japan ; \\ "PRESTO, Japan Science and Technology Agency, 4-1-8 Honcho Kawaguchi, Saitama, Japan
}

Correspondence should be addressed to T.H., Tel:+81-43-290-2926. Fax:+81-43-290-2925. E-mail: hoshi@p.chiba-u.ac.jp

\begin{abstract}
Not a single water molecule but a group of water molecules frequently play an important role to functionalize the enzymatic reaction of proteins. In this study, we carried out molecular dynamics (MD) simulations for 4 types of myosin-nucleotide complexes; the ATP and/or ADP/P $\mathrm{P}_{\mathrm{i}}$-bound myosins with the cleft open and/or close conformations. Computational results show a keen role of water molecules those are regulated by a salt-bridge formed between Arg238 in Switch I and Glu459 in Switch II. Before the ATP hydrolysis, Arg238 and Glu459 hold a lytic water near ATP- $\gamma$ phosphate and close the salt-bridge to inhibit any other water to enter the ATPase pocket except one water that controls the orientation of the lytic water facing to ATP. Once the salt-bridge is opened after ATP hydrolysis, a large number of water molecules are circulating in the ATPase pocket. Our results demonstrate that the access of water molecules is precisely controlled in the respective reaction step of the ATP hydrolysis cycle.
\end{abstract}

Key words water molecules, molecular dynamics, hydrolysis, salt bridge, ATPase, myosin 


\section{Introduction}

Water molecules play an essential role in many aspects of biological phenomena and frequently dominate the function of proteins. Many previous theoretical studies paid much attention to only a few critical water molecules those stayed at the specific position inside an enzyme; i.e., the lytic water in serine proteases [1-4] or the stabilizing water in proteins [5]. In contrast, some of recent studies addressed the dynamic motion of a group of water molecules or the special arrangement of a group of waters. For example, the folding of protein largely depends on the dynamics of waters [6-8], transmembrane proteins requires the formation of water chain inside it [9,10], and the action of ion channel is caused by the flow of waters [11]. In the present work, we focused on the circulation of waters in ATPase pocket and examine the accessibility of waters to the active site in terms of the conversion of the protein conformations.

Myosin is a motor protein that causes contractile movement of muscles and its function is regulated by the hydrolysis of ATP. Myosin holds ATP and hydrolyzes it to produce adenosine diphosphate (ADP) and inorganic phosphate $\left(\mathrm{P}_{\mathrm{i}}\right)$. These products are released in the subsequent step of the hydrolysis reaction and myosin returns to the substrate-free state. Repeating this process, myosin filaments slide on actin filament. Three dimensional structures of myosins were already determined by X-ray crystallographic studies [12-16]. According to these studies, nucleotide (ATP or ADP) and actin binding sites were found to be located in the globular head of myosin, called subfragment 1 or S1. The nucleotide binding site (called the ATPase pocket) is surrounded by three loop structures; the P-loop, the switch I loop, and the switch II loop. Many experiments by the site-directed mutation approach suggested that several amino acid residues at the ATPase pocket played an important role in the hydrolysis of ATP [17-21]. Namely, the saltbridge between Arg238 in Switch I and Glu459 in Switch II was expected to essentially support the ATP hydrolysis. In addition, Onishi et. al. reported that Ser237 and Gly457 played an important role in holding the lytic water [22, 23].

Bagshaw et. al. $[24,25]$ proposed the following seven steps on the mechanism of the binding and the hydrolysis of ATP by myosin. Step1: An ATP is bound to myosin. Step2: The bound ATP induces the conformational change of myosin. Step3: The ATP is hydrolyzed by myosin. Step4: myosin changes its own conformation to release $\mathrm{P}_{\mathrm{i}}$. Step5: $\mathrm{P}_{\mathrm{i}}$ is released from myosin. Step6: A myosin-ADP complex is formed. Step7: ADP is released from myosin and the conformation of myosin returns to its original state. Sutoh et. al. showed that a large angle change of the lever arm was necessary for the stroke motion from the step 1 to step 2 [26].

In this work, we focused on the relationship between the behavior of water molecules and the structural change of switch I and II of myosin . Based on the ATP hydrolysis cycle of myosin, we made 4 kinds of model structures on myosin nucleotide complexes (model 1: ATP-bound cleft open myosin, which corresponds to step1; model 2: ATPbound cleft close myosin, which corresponds to step2; model 3: ADP/ $\mathrm{P}_{\mathrm{i}}$-bound cleft close myosin, which corresponds to step3, model 4: $\mathrm{ADP} / \mathrm{P}_{\mathrm{i}}$-bound cleft open myosin, which corresponds to step4) and performed molecular dynamics 
(MD) simulations for each model structure.

\section{Computational Section}

2.1. Construction of the initial structures

myosins complexed with ATP and/or ADP/ $\mathrm{P}_{\mathrm{i}}$. The initial structures of these complexes were constructed, based on the $\mathrm{X}$-ray crystallographic structures of myosin-ATP $\gamma \mathrm{S}$ complex (the template for cleft open myosin: the Protein Data Bank (PDB) entry code is $1 \mathrm{MMG}$ [27]) and myosin-ADP/VO $/ \mathrm{VO}_{4}$ complex (the template for cleft close myosin: the PDB entry code is 1VOM [14]). Because many amino acid residues were absent in the coordinate data of these X-ray structures, we repaired them by adding the lacking amino acid residues (1MMG: Met1, Gln204-Ser208, Trp501Gly507, Lys622-Asn626) (1VOM:Ala205-Ser208, Asn711, Ala716-Ser719, Asp724-Leu730), using the homology module of Insight II (Insight II, version 95.0, Molecular Simulations Inc., San Diego).

In order to make a model structure for the cleft-open myosin complexed with ATP, we replaced the ATP $\gamma \mathrm{S}$ part of the X-ray structure of $1 \mathrm{MMG}$ [27] with ATP and a lytic-water, the atom positions of ATP and a water were determined by referring the previous quantum chemical (QC) calculation [28]. To make a model structure for the cleft-close myosin complexed with $\mathrm{ATP}$, the $\mathrm{ADP} / \mathrm{VO}_{4}$ part of the X-ray structure of $1 \mathrm{VOM}$ [14] was replaced with ATP and a lytic-water. For the cleft-close myosin complexed with $\mathrm{ADP} / \mathrm{P}_{\mathrm{i}}$, the $\mathrm{ADP} / \mathrm{VO}_{4}$ part of the structure of $1 \mathrm{VOM}$ was replaced by $\mathrm{ADP} / \mathrm{P}_{\mathrm{i}}$, and for the cleft-open myosin complexed with $\mathrm{ADP} / \mathrm{P}_{\mathrm{i}}$, the ATP $\gamma \mathrm{S}$ part of the structure of $1 \mathrm{MMG}$ was replaced by $\mathrm{ADP} / \mathrm{P}_{\mathrm{i}}$.

\subsection{Calculations}

Molecular mechanics (MM) potential energy minimizations and MD simulations were carried out using the program package AMBER (version 7.0, University of California) [29]. Calculations were performed using the ff99 force field. The system was solvated in a rectangular box of about $110 \AA$ x $90 \AA$ x $80 \AA$ in size, and about 20,000 TIP3P water molecules [30] were generated in the box. The periodic boundary condition was applied and the pressure was kept constant at $1.0 \mathrm{~atm}$. The temperature was kept constant according to Berendsen's algorithm with a coupling time of 0.2 ps [31]. The SHAKE method was used to simplify the calculation [32]. The nonbonded interactions were calculated by applying a cut off of $15 \AA$. The integration time step of the MD simulations was $1 f s$.

Potential energy minimizations were firstly performed on the initial structures. In the potential energy minimizations, the steepest descent method was used for the early cycles and then the conjugate gradient method was used later. MD simulations were performed, taking careful consideration on the temperature. That is, preliminary 10 ps MD simulations were executed at $300 \mathrm{~K}$ only for the solvent water molecules with the enzyme, nucleotide, and crystal water molecules fixed. Next, the temperature of all systems was gradually increased by heating up to $300 \mathrm{~K}$ for the first $70 \mathrm{ps}$, and then kept at $300 \mathrm{~K}$ for the next $1 \mathrm{~ns}$. The trajectories for $1 \mathrm{~ns}$ simulation at $300 \mathrm{~K}$ were 
considered to be the most probable structure under physiological conditions and were analyzed in detail.

\section{Results and discussion}

Figs. 1(a)-(d) show the last structures of the ATPase pocket obtained from MD simulations for four types of myosin nucleotide complexes. Although these are snapshot at $1 \mathrm{~ns}$, they all demonstrate typical conformations seen in the respective simulation. The averaged structures for 500-1000 ps are also depicted in Figs. 1(a')-(d') to confirm the adequateness of those snapshot structures. A prominent difference among those four structures is the number of waters occupying the ATPase pocket; 10 waters for ATP-bound cleft-open myosin, 2 for ATP-bound cleft-close myosin, 1 for $\mathrm{ADP} / \mathrm{P}_{\mathrm{i}}$-bound cleft-close myosin, 5 for $\mathrm{ADP} / \mathrm{P}_{\mathrm{i}}$-bound cleft-open myosin. It is natural that more water molecules appear inside the ATPase pocket of the cleft-open myosin than the cleft-close one both for the ATP-bound and the $\mathrm{ADP} / \mathrm{P}_{\mathrm{i}}$-bound cases. In both of the cleft-open and close states, less water molecules enter the ATPase pocket of ATP/ $\mathrm{P}_{\mathrm{i}}$-bound myosin compared to the ATP-bound one. The hydrolysis conversion of ATP to ADP/ $\mathrm{P}_{\mathrm{i}}$ causes the increase of the volume occupied by the nucleotide and phosphate, which decreases the room for waters to stay. In the cleft-close myosin, the guanidino group of Arg238 makes a hydrogen bond with the carbonyl group of Glu459, in which the distance between $\operatorname{Arg} 238-\mathrm{N} \eta$ and Glu459-O $\varepsilon 1$ is $2.9 \AA$. The atom for the hydrogen bond acceptor sometimes switched from Glu459-Oe1 to Glu459-Oe2 (See d2 and d3 of Fig. 2). Namely the carbonyl group of Glu459 can changes its orientation while keeping the salt bridge. This change of the hydrogen bond acceptor was seen a few times in $1 \mathrm{~ns}$ MD simulation. Similar change in the orientation of carbonyl group of Glu459 was observed in the ADP/ $\mathrm{P}_{\mathrm{i}}$-bound cleft-close myosin as shown in $\mathrm{d} 2$ and $\mathrm{d} 3$ of Fig. 3 . After the ATP hydrolysis to ADP/ $\mathrm{P}_{\mathrm{i}}$, the product $\mathrm{P}_{\mathrm{i}}$ is attracted by the guanidino group of $\mathrm{Arg} 238$. The distances between $\mathrm{P} \gamma$ atom and two $\mathrm{N}$ atoms of $\operatorname{Arg} 238$ in the ADP/ $\mathrm{P}_{\mathrm{i}}$-bound cleft-open myosin become the shortest among four types of simulation models as shown in $\mathrm{d} 4$ and $\mathrm{d} 5$ of Fig. 4. This attraction would enhance the detachment of $\mathrm{P}_{\mathrm{i}}$ from the pocket.

The water molecule for ATP hydrolysis (lytic water) shows a large difference in its movement between in the cleft open and the cleft close ATP-bound myosins. Initially, the myosin-ATP complex had been modeled with a water molecule set at the vicinity of ATP in both cases. When the cleft was opened, the water molecule still stayed at the adequate position for ATP hydrolysis for 1-500 ps. However the water escaped from the pocket at $730 \mathrm{ps}$, and the distance between the water and P $\gamma$ of ATP became about $11 \AA$ at 1 ns (See d1 of Fig. 5). On the other hand, when the cleft was closed, the distance between the water and P $\gamma$ of ATP was kept at about $3.0 \AA$ and the water stayed in the ATPase pocket for $1 \mathrm{~ns}$ (See d1 of Fig. 2). These results indicate that the open and close of the cleft, which corresponds to the formation and the break of a salt-bridge between the side chains of $\operatorname{Arg} 238$ and Glu459, is responsible for the reaction probability of the ATP hydrolysis by myosin, because the lytic water is required to approach P $\gamma$ of ATP up to $2.2 \AA$ according to the previous QC calculation [28]. In addition, these results give us one 
possible hypothesis that this salt-bridge has an important effect on the circulation of waters in the ATPase pocket. Hence, in order to examine if there were any differences in the movements of other waters between the cleft-open and the cleft-close states of myosin, we investigated the behavior of water molecules through the following procedure. A water molecule is selected. Three distances from $\mathrm{O}$ of the water to $\mathrm{C} \delta$ of Glu459, to $\mathrm{C} \zeta$ of $\operatorname{Arg} 238$, and to $\mathrm{O} \beta$ of ATP/ADP are measured. If all three distances are within $8.0 \AA$, the water is assumed to be in the ATPase pocket. The measurement of three distances is executed for all coordinations of $1 n s$ simulation and is performed for all water molecules. (ATP-bound cleft open myosin: 20,547 waters, ATP-bound cleft close myosin: 20,338, ADP/ $\mathrm{P}_{\mathrm{i}}$-bound cleft close myosin: 20,240, ADP/ $\mathrm{P}_{\mathrm{i}}$-bound cleft open myosin: 20,447). The appearance of water molecules satisfying the above criteria is shown through the simulation in Fig. 6.

Fig. 6 indicates that 34 waters entered in the ATPase pocket for the ATP-bound cleft open myosin (Fig. 6a), while only 4 waters came in the ATPase pocket for the ATP-bound cleft close myosin (Fig. 6b). The average number of waters inside the ATPase pocket during the simulations are 6.5 and 2.4, respectively. In Fig. 6a, the water (water no.2) that left from the ATPase pocket at about $750 \mathrm{ps}$ was the water set at the hydrolysis position at the beginning of the simulation. Another water (water no.1) was kept inside the ATPase pocket for 1ns (momentarily disappeared from the ATPase pocket at $810 \mathrm{ps}$ ). The hydrogen bond network around this water molecule at $1 n s$ is shown in Fig. 7a. This water no.1 appears to be stably kept in the ATPase pocket by forming hydrogen bonds among Arg238, Ser237, and ATP.

In the X-ray crystal structure of the $\mathrm{MgADP} / \mathrm{BeF}_{\mathrm{x}}$-bound complex that mimics the pre-hydrolysis state of the myosin-ATP complex [13], a water molecule makes hydrogen bonds with a fluoride of $\mathrm{BeF}_{\mathrm{x}}$ and with the main-chain carbonyl oxygen of Ser237 (Ser246 in the crystallographic data: PDB\# 1MMD). The water molecule also interacts electrostatically with the guanidino group of Arg238. Based on these experimental findings, Onishi et. al. proposed that Arg238 (Arg247 in their report) indirectly interacted with $\gamma$-phosphate via this water and, for this reason, Arg238 was important for the nucleotide binding and the initiation of the cleft close motion [22,23]. It is interesting to see that our simulation is quite compatible with their proposal except for the following one point. In our simulation, the water molecule is kept by Arg238, ATP, and Ser236 (Fig. 7a) while the water molecule is kept by Arg238, ATP, and Ser237 in their proposal.

In the ATP-bound cleft close myosin, a water (water no.1) was stably kept in the ATPase pocket for 1 ns. Another water (water no.2) entered at $60 \mathrm{ps}$ and repeated the in and out motions until $250 \mathrm{ps}$, and after that, the water was stably kept in the pocket (Fig. 6b). The locations of these two waters at $1 \mathrm{~ns}$ are shown in the schematic drawing of the inside of the ATPase pocket in Fig. 7b. It is confirmed from this figure that the water no.2 is kept in the ATPase pocket by forming hydrogen bonds with $\mathrm{O}$ of Gly457 and $\mathrm{O} \varepsilon 1$ of Glu459 and that the water no.1 (lytic water) is kept 
by forming hydrogen bonds with $\mathrm{O} \gamma 3$ of ATP and $\mathrm{O}$ of the water no.2. Namely, when the cleft is closed preceding the ATP hydrolysis, only a few water molecules but the lytic water can come into the ATPase pocket, and one of these water molecules stably stays in the ATPase pocket. This water molecule makes a firm hydrogen bond with the lytic water and supports the lytic water to be in the proper orientation when the lytic water attacks ATP.

In the X-ray crystallographic structure of the MgADP-vanadate-bound complex that mimicked the transition state of the ATP-bound myosin [14], a water molecule was detected in the ATPase pocket. The water molecule was assumed to make hydrogen bonds with the main-chain carbonyl oxygen of Gly457 (Gly468 in the crystallographic data: PDB\# 1VOM) and with one of the two oxygen atoms in the carboxyl group of Glu459. Based on these findings, Onishi et. al. also proposed that this water molecule played an important role in making an optimal enzymatic conformation for hydrolysis, that is, this water assisted the lytic water to be in an adequate position for a nucleophilic attack on the $\gamma$-phosphate $[12,13]$. A satisfactory consistency is seen between our results and their proposal. Hence these two water molecules are considered to be strongly concerned in the ATP hydrolysis in myosin. There are, however, a slight difference between our simulation result and their proposal. They proposed one $\mathrm{H}$ of the lytic-water made a hydrogen bond with $\mathrm{O}$ of the water no.2, the other $\mathrm{H}$ of the lytic-water made a hydrogen bond with $\mathrm{O}$ of Ser237, and O of the lytic water was oriented toward P $\gamma$ of ATP. In our previous computational study on the ATP hydrolysis mechanism of myosin, one $\mathrm{H}$ of the lytic water was transferred to $\mathrm{O} \gamma 3$ of ATP and the remaining $\mathrm{OH}$ group of the lytic water attacked to P $\gamma$ of ATP in the transition state [28]. This mechanism was shown to be common to the GTP-> GDP conversion in G proteins $[1,2,4]$. In the present MD simulation, one $\mathrm{H}$ of the lytic water makes a hydrogen bond with $\mathrm{O}$ of the water no.2, while the other $\mathrm{H}$ of the lytic water makes hydrogen bond with $\mathrm{O} \gamma 3$ of ATP (Fig. 7b). Hydrogen bond network around the lytic water seen in the MD simulation is more similar to the results of the previous QM calculation [28] than the proposal of Onishi et. al. [22].

In the simulation of the $\mathrm{ADP} / \mathrm{P}_{\mathrm{i}}-$ bound cleft close myosin, the number of water molecules approaching to the ATPase pocket was only two (Fig. 6c). This number is the least among four simulations. In addition, both water molecules did not stay in the ATPase pocket for a long time. Hence, the average number of waters inside the ATPase pocket is only 0.3 . On the other hand, in the simulation of the $\mathrm{ADP} / \mathrm{P}_{\mathrm{i}}$-bound cleft open myosin, the number of water molecules circulating in the ATPase pocket was 40 (Fig. 6d). This number is the largest among four simulations. All these 40 water molecules did not stay in the ATPase pocket for a long time. The average time for staying in the ATPase pocket was only 20 ps. Hence, the water molecules in the ATPase pocket are very rapidly exchanged in the last model. Accordingly, the average number of waters inside the ATPase pocket during the simulation is not so large; 3.0. We speculate that this water circulation is effective for getting rid of reaction products from the pocket. 
In MD simulation for the ATP-bound cleft open myosin, the distances between Arg238 and Glu459 (d2, d3 in Fig. 5) and between ATP and Glu459 (d6, d7 in Fig. 5) fluctuated in a wide range. On the other hand, the distance between ATP and Arg238 (d4, d5 in Fig. 5) almost kept constant. These results indicate that the movement of Glu459 (Switch II) is more important than that of Arg238 (Switch I) for the formation of the salt bridge. This result is consistent with the difference between two myosin conformations in the X-ray crystallographic data. A comparison of crystal structures of myosin between $\mathrm{ADP} / \mathrm{VO}_{4}$ (transition state analogue) and $\mathrm{ADP} / \mathrm{BeF} \mathrm{F}_{\mathrm{x}}(\mathrm{ATP}$ analog) suggested a large structural change for Switch II, but little for Switch I [23]. In addition, these our results are compatible with previous reports $[33,34,35]$

Many kinetic studies indicate that $\mathrm{P}_{\mathrm{i}}$ is released prior to ADP during the contractile cycle and $\mathrm{P}_{\mathrm{i}}$ would be ejected from the back door [36]. In our simulation, the circulation of a lot of water molecules is induced by the break of the salt-bridge between Arg238 and Glu459. Water molecules enter in the ATPase pocket and they bounce inside and some of them happen to leave for outside. In the snapshot structure at the final point of our MD simulation, the distance between Gly457-C $\alpha$ and ATP-P $\alpha$ atoms is $12.2 \AA$ in the ATP-bound cleft-open myosin (Fig. 1a). This distance increases to $13.1 \AA$ in the $\mathrm{ADP} / \mathrm{P}_{\mathrm{i}}$-bound cleft-open myosin (Fig. 1d). Further, the distance between $\mathrm{P} \gamma$ and $\mathrm{P} \beta$ atoms in the $\mathrm{ADP} / \mathrm{P}_{\mathrm{i}}$-bound myosin increased from $4.3 \AA$ to $4.8 \AA$ in accordance with the conversion from the close to the open conformation of the cleft. These changes in distances intimate the movement of ADP toward the front door and the opposite movement of $\mathrm{P}_{\mathrm{i}}$ to the back door in the $\mathrm{ADP} / \mathrm{P}_{\mathrm{i}}$-bound cleft-open myosin. This result is completely consistent with the findings by Lawson et al. [37]. Accordingly, we speculate that the circulation of a great number of water molecules is one of the driving forces for releasing $\mathrm{P}_{\mathrm{i}}$ and ADP from myosin and a long time MD simulation can demonstrate the release motions of ADP and $\mathrm{P}_{\mathrm{i}}$.

\section{Conclusion}

MD simulations for 4 types of myosin-nucleotide complexes provided the following findings. [1] When the cleft is opened in the ATP-bound myosin, a water molecule is possible to stay in the ATPase pocket by forming hydrogen bonds with Arg238, Ser236 and ATP. However, no lytic water steadily stays in the ATPase pocket. [2] When the cleft is closed in the ATP-bound myosin, two waters stably stay in the ATPase pocket. One is the lytic water, and the other is also essential for supporting the lytic water to be in a proper orientation to attack ATP. [3] When the cleft is closed in the ADP/ $\mathrm{P}_{\mathrm{i}}$-bound myosin, water molecules scarcely access to the ATPase pocket. [4] When the cleft is opened in the ADP/ $/ \mathrm{P}_{\mathrm{i}}$-bound myosin, a lot of water molecules circulate in the ATPase pocket and these water molecules are very rapidly exchanged. The close of the cleft (the formation of a salt-bridge between Arg238 and 
Glu459) promotes the ATP hydrolysis of myosin and the open of the cleft enhances the release of $\mathrm{P}_{\mathrm{i}}$ and ADP from myosin.

\section{Acknowledgement}

A part of this work was supported by PRESTO, Japan Science and Technology Agency. This work was partly supported by Grant-in Aid for Center of Excellence (COE) research from the Ministry of Education, Sciences, Sport, and Culture, Japan. A part of this work was executed by the super computer Vpp700E in RIKEN. 


\section{References}

[1] N. Futatsugi, M. Hata, T. Hoshino and M. Tsuda, Biophys. J., 77 (1999) 3287.

[2] Y. Hirano, M. Hata, T. Hoshino and M. Tsuda, J. Phys. Chem. B, 106 (2002) 5788.

[3] Y. Fujii, M. Hata, T. Hoshino and M. Tsuda, J. Phys. Chem. B, 106 (2002) 9687.

[4] D. Katagiri, M. Hata, T. Itoh, S. Neya and T. Hoshino, J. Phys. Chem. B, 107 (2003) 3278.

[5] N. Okimoto, T. Tsukui, K. Kitayama, M. Hata, T. Hoshino and M. Tsuda, J. Am. Chem. Soc., 122 (2000) 5613.

[6] Y. M. Rhee, E. J. Sorin, G. Jayachandran, E. Lindahl and V. S. Pande, Proc. Natl. Acad. Sci. U.S.A., 101 (2004) 6456.

[7] Y. Harano and M. Kinoshita, Chem. Phys. Lett., 399 (2004) 342.

[8] D. Russo, G. Hura and T. Head-Gordon, Biophys. J., 86 (2004) 1852.

[9] K. Murata, Y. Fujii, N. Enomoto, M. Hata, T. Hoshino and M. Tsuda, Biophys. J., 79 (2000) 982.

[10] Y. S. Lee and M. Krauss, J. Am. Chem. Soc., 126 (2004) 2225.

[11] W. Im and B. Roux, J. Mol. Biol., 319 (2002) 1177.

[12] I. Rayment, W. R. Rypniewski, K. Schmidt-Base, R. Smith, D. R. Tomchick, M. M. Benning, D. A. Winkelmann, G. Wesenberg and H. M. Holden, Science, 261 (1993) 50.

[13] A. J. Fisher, C. A. Smith, J. B. Thoden, R. Smith, K. Sutoh, H. M. Holden and I. Rayment, Biochemistry, 34 (1995) 8960.

[14] C.A. Smith and I. Rayment, Biochemistry, 35 (1996) 5404.

[15] R. Dominguez, Y. Freyzon, K. M. Trybus and C. Cohen, Cell, 94 (1998) 559.

[16] A. Houdusse, V. N. Kalabokis, D. Himmel, A. G. Szent-Gyorgyi and C. Cohen, Cell, 97 (1999) 459.

[17] H. Onishi, S. Kojima, K. Katoh, K. Fujiwara, H. M. Martinez and M. F. Morales, Proc. Natl. Acad. Sci. U.S.A.,95 (1998) 6653.

[18] H. Onishi, M. F. Morales, S. Kojima, K. Katoh and K. Fujiwara, Biochemistry, 36 (1997) 3767.

[19] N. Sasaki, T. Shimada and K. Sutoh, J. Biol. Chem. 273 (1998) 20334.

[20] X. D. Rhodes, T. E. Li, R. Ikebe, T. Kambara, H. D. White and M. Ikebe, J. Biol. Chem., 273 (1998) 27404.

[21] M. Furch, S. Fujita-Becker, M. A. Geeves, K. C. Holmes and D. J. Manstein, J. Mol. Biol., 290 (1999) 797.

[22] H. Onishi, T. Ohki, N. Mochizuki and M. F. Morales, Proc. Natl. Acad. Sci. U.S.A.,99 (2002) 15339.

[23] H. Onishi, N. Mochizuki and M. F. Morales, Biochemistry, 43 (2004) 3757.

[24] C. R. Bagshaw and D. R. Trentham, Biochem. J., 141 (1974) 331.

[25] C. R. Bagshaw, J. F. Eccleston, F. Eckstein, R. S. Goody, H. Gutfreund and D. R. Trentham, Biochem. J., 141 (1974) 351. 
[26] Y. Suzuki, T. Yasunaga, R. Ohkura, T. Wakabayashi and K. Sutoh, Nature, 396 (1998) 380.

[27] A. M. Gulick, C. B. Bauer, J. B. Thoden and I. Rayment, Biochemistry, 36 (1997) 11619.

[28] N. Okimoto, K. Yamanaka, J. Ueno, M. Hata, T. Hoshino and M. Tsuda, Biophys. J., 81 (2001) 2786.

[29] D. A. Perlman, D. A. Case, J. W. Caldwell, W. S. Ross, T. E. Cheatham III, S. DeBolt, D. Ferguson, G. Seibel and

P. A. Kollman, Comp. Phys. Commun., 91 (1995) 1.

[30] W. L. Jorgensen, L. Chandrasekhar and J. D. Madura, J. Chem. Phys., 79 (1983) 926.

[31] H. J. Berendsen, J. P. Postma, W. F. van Gunsteren, A. DiNola and J. R. Haak, J. Chem. Phys., 81 (1984) 3684.

[32] J. P. Ryckaert, G. Ciccotti and H. J. Berendsen, J. Comput. Phys., 23 (1977) 327.

[33] T. J. Minehardt, N. Marzari, R. Cooke, E. Pate, P. A. Kollman and R. Car, Biophys. J., 82 (2002) 660.

[34] G. Li and Q. Cui, Biophys. J., 86 (2004) 743.

[35] G. Li and Q. Cui, J. Phys. Chem. B, 108 (2004) 3342.

[36] R. G. Yount, J. D. Lawson and I. Rayment, Biophys. J., 68 (1995) 44s.

[37] J.D. Lawson, E. Pate, I. Rayment and R. G. Yount, Biophys. J., 86 (2004) 3794. 


\section{Figure legends}

Figure 1. Structures inside the ATPase pocket. (a) ATP-bound cleft-open myosin. (b) ATP-bound cleft-close myosin. (c) $\mathrm{ADP} / \mathrm{P}_{\mathrm{i}}$-bound cleft-close myosin. (d) $\mathrm{ADP} / \mathrm{P}_{\mathrm{i}}$-bound cleft-open myosin. Adenosine phosphates are indicated by sticks. Four residues; Ser236, Asp238, Glu457, Glu459, and water molecules are shown by the ball and stick representation. The distances are in unit of $\AA$. The yellow, blue, and pink regions indicate switch I, switch II, and Ploop, respectively. (a'), (b'), (c'), (d') are the averaged structures for 500-1000 ps.

Figure 2. ATP-bound cleft close myosin. The distance d1 between $\mathrm{O}$ of the lytic water and P $\gamma$ of ATP is kept at about $3.3 \AA$ for $1 n s$ MD simulation, and the minimum distance is $2.86 \AA$. The distances $\mathrm{d} 2$ and $\mathrm{d} 3$ are hardly changed for 1

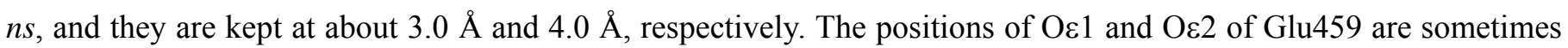
interchanged. The distance $\mathrm{d} 4$ is kept at about $4.0 \AA$ and $\mathrm{d} 5$ is kept at about $6.0 \AA$. The distance d6 fluctuates between 3.5 and 7.5 $\AA$, and $\mathrm{d} 7$ fluctuates between 5.5-7.5 $\AA$. The distance $\mathrm{d} 8$ is kept at about $3.0 \AA$ for $1 \mathrm{~ns}$ MD simulation.

Figure 3. ADP/ $\mathrm{P}_{\mathrm{i}}$-bound cleft close myosin. The distance $\mathrm{d} 1$ between $\mathrm{P} \gamma$ of $\mathrm{P}_{\mathrm{i}}$ and $\mathrm{O} \beta 3$ of ADP is kept at about $2.3 \AA$ for $1 n s$ simulation. The distance $\mathrm{d} 2$ is kept at about $2.7 \AA$ and $\mathrm{d} 3$ is changed between $3.2-5.0 \AA$ for $0-320$ ps. At 320 ps, the positions of $\mathrm{O} \varepsilon 1$ and $\mathrm{O} \varepsilon 2$ of Glu459 are interchanged and then $\mathrm{d} 3 \mathrm{is}$ kept at about 2.7 for $320-750 \mathrm{ps}$ and $\mathrm{d} 2$ fluctuated between 3.2-5.0 A. At $750 \mathrm{ps}$, the positions of $\mathrm{O} \varepsilon 1$ and $\mathrm{O} \varepsilon 2$ of Glu459 are interchanged again. As for the distances of $\mathrm{d} 4$ and $\mathrm{d} 5, \mathrm{~d} 4$ is kept at about $3.8 \AA$ and $\mathrm{d} 5$ is kept at about $5.8 \AA$. Behavior of $\mathrm{d} 6$ and $\mathrm{d} 7 \mathrm{resembles}$ those of $\mathrm{d} 2$ and $\mathrm{d} 3$. The distance $\mathrm{d} 6$ is kept in about 4.0-6.5 $\AA$ and $\mathrm{d} 7 \mathrm{is} \mathrm{kept} \mathrm{in} \mathrm{5.5-8.5} \AA$ for 0-320 ps and 750-1000 ps. From $320 \mathrm{ps}$ to $750 \mathrm{ps}$, the positions of $\mathrm{O} \varepsilon 1$ and $\mathrm{O} \varepsilon 2$ are interchanged and, $\mathrm{d} 6$ is kept in $5.5-8.5 \AA$ and $\mathrm{d} 7$ is kept in 4.0-6.5 $\AA$. The distance of $\mathrm{d} 8$ is kept at about $3.4 \AA$ for $1 \mathrm{~ns}$.

Figure 4. ADP/ $\mathrm{P}_{\mathrm{i}}$-bound cleft open myosin. The distance between $\mathrm{P} \gamma$ of $\mathrm{P}_{\mathrm{i}}$ and $\mathrm{O} \beta 3$ of ADP (d1) is kept at about $3.4 \AA$ for $1 n s$. The distances $\mathrm{d} 2$ and $\mathrm{d} 3$ are changed in a wide range (4.5-10 $\AA$ ) for $1 n s$. The distances $\mathrm{d} 4$ and $\mathrm{d} 5 \mathrm{move}$ around $3.2 \AA$ and $5.2 \AA$, respectively. The distances $\mathrm{d} 6$ and $\mathrm{d} 7$ also fluctuate in a wide range $(5.0-11.0 \AA)$. The distance $\mathrm{d} 8$ expands from 6.0 to $10.0 \AA$

Figure 5. ATP-bound cleft open myosin. (a) Initial structure at the inside of ATPase pocket. (b)-(f) The distances of $\mathrm{d} 1-\mathrm{d} 8$ are plotted against the simulation time. The distance $\mathrm{d} 1$ indicates that the lytic water starts to get away from $\mathrm{P} \gamma$ of ATP at $500 \mathrm{ps}$ and the distance becomes $11.0 \AA$ at $1 \mathrm{~ns}$. The distances $\mathrm{d} 2$ and $\mathrm{d} 3$ are changed in a wide range in 1 
$n s ; \mathrm{d} 2: 2.6-8.5 \AA, \mathrm{d} 3: 2.7-9.0 \AA$. In addition, the positions of $\mathrm{O} \varepsilon 1$ and $\mathrm{O} \varepsilon 2$ of Glu459 are sometimes interchanged (220-250 ps, 400-500 ps). As for the distances of $\mathrm{d} 4$ and $\mathrm{d} 5$, they are seldom changed in $1 \mathrm{~ns}$ MD simulation; $\mathrm{d} 4$ is kept at about $6.0 \AA$, d5 is kept at about $8.0 \AA$. Behaviors of the d6 and d7 resemble those of d2 and d3. The fluctuations are in a wide range in $1 \mathrm{~ns}$ simulation and the positions of $\mathrm{d} 5$ and $\mathrm{d} 6$ are interchanged at 220-250 ps and 360-480 ps. The distance $\mathrm{d} 8$ changes between 5 and $11 \AA \AA$ during $1 n s$ simulation.

Figure 6. Presence of water molecules in access to the ATPase pocket. One horizontal line indicates that one water molecule is present in the ATPase pocket at that time. This graph provides information on the number of water molecules in the ATPase pocket at any time point and on the lifetime of each water in access. (a)Water molecules in the ATPase pocket for the ATP-bound cleft open myosin model. (b) Water molecules in the ATPase pocket for the ATP-bound cleft close myosin model. (c) Water molecules in the ATPase pocket for the ADP/ $\mathrm{P}_{\mathrm{i}}$-bound cleft close myosin model. (d) Water molecules in the ATPase pocket for the ADP/ $\mathrm{P}_{\mathrm{i}}$-bound cleft open myosin model.

Figure 7. Schematic representation of the ATP-bound myosin in the cleft-open state (a) and the cleft-close state (b). Hydrogen bonds are indicated by dashed lines. 

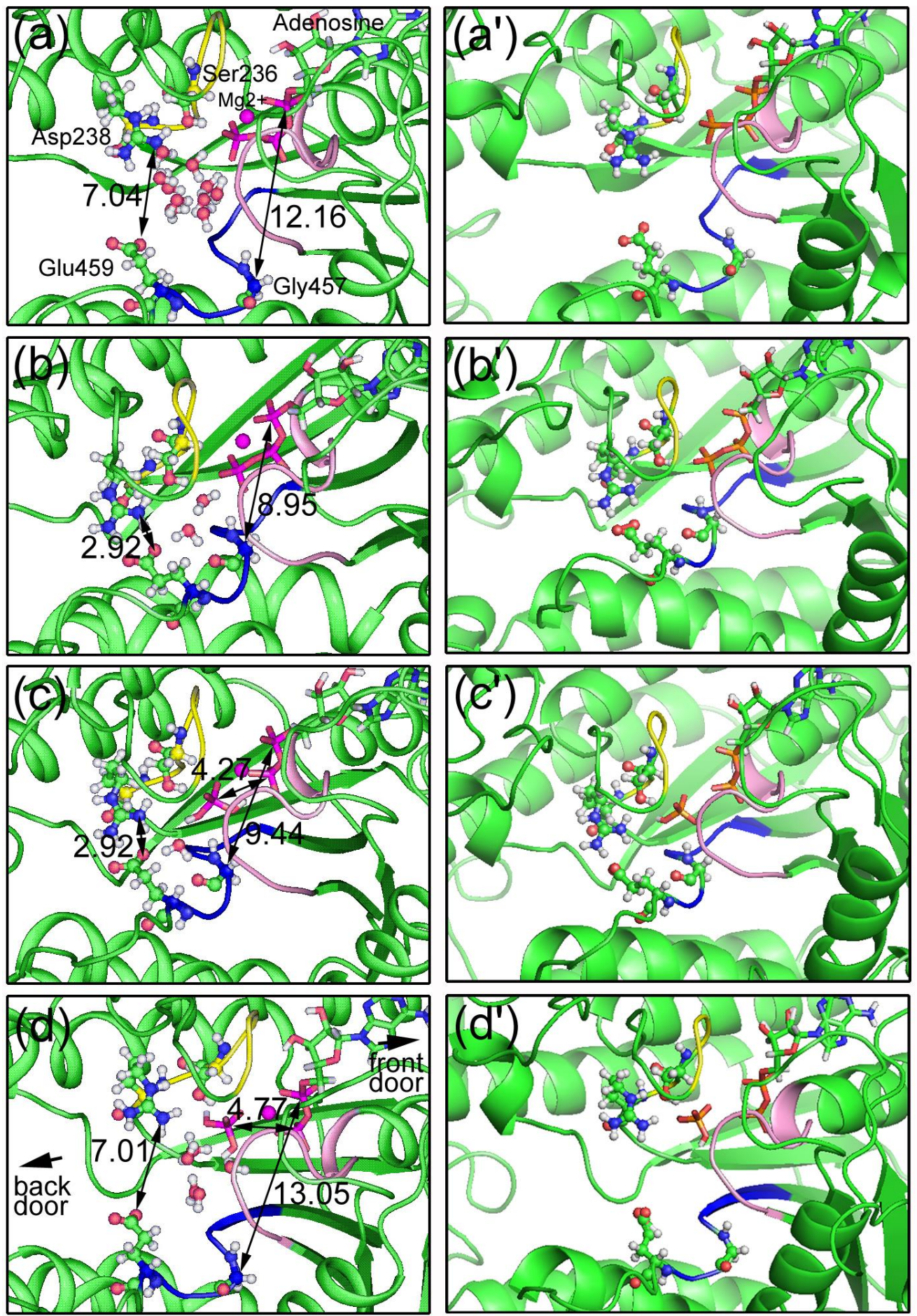

Figure 1

T. Hoshino et al. Behavior of water molecules in ATPase pocket of myosin 
(a)

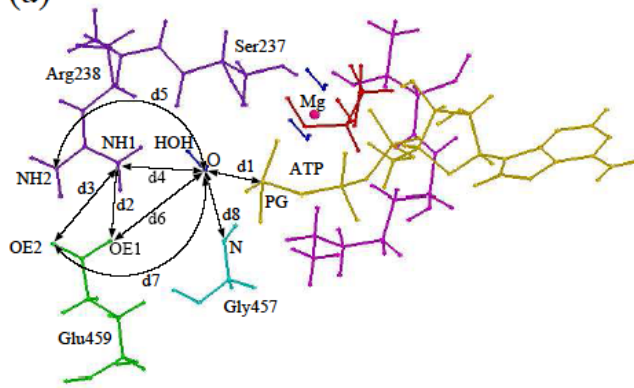

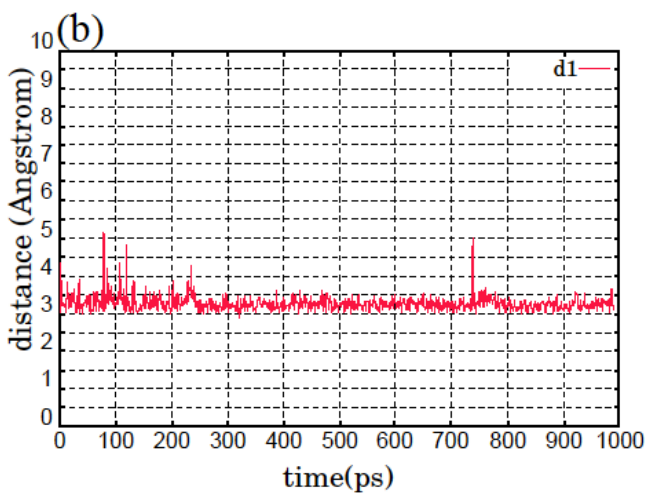
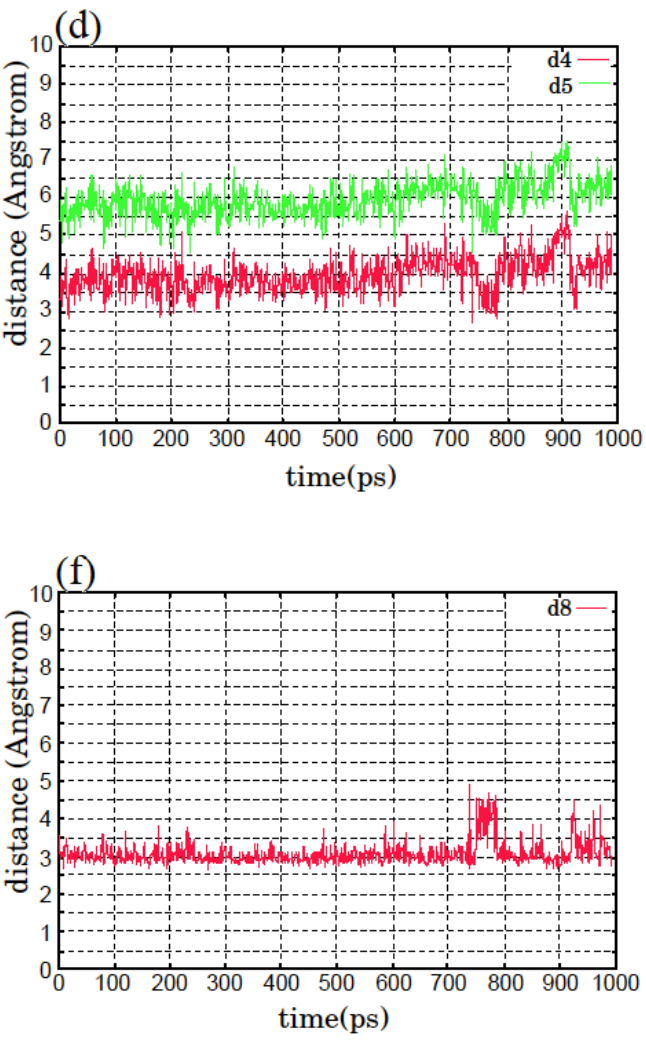

Figure 2

\section{T. Hoshino et al. Behavior of water molecules in ATPase pocket of myosin}


(a)

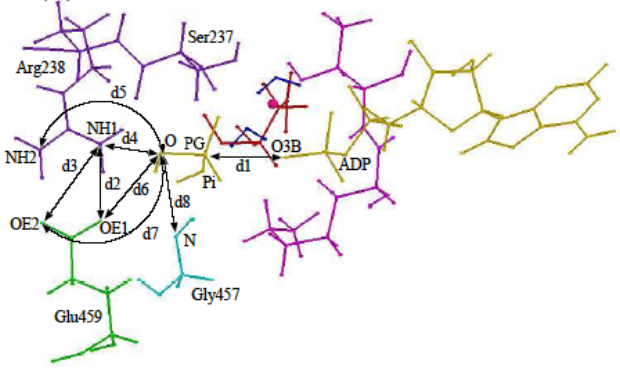

10 (b)

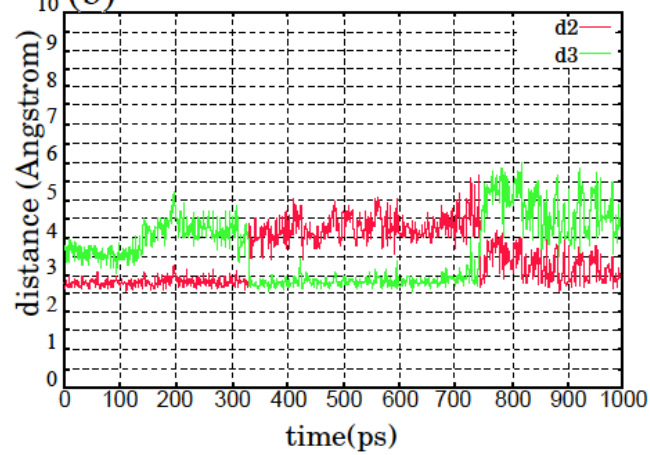

(d)

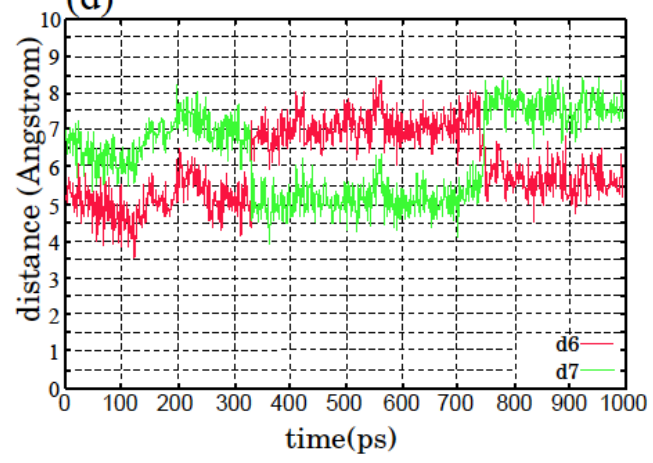

(b)

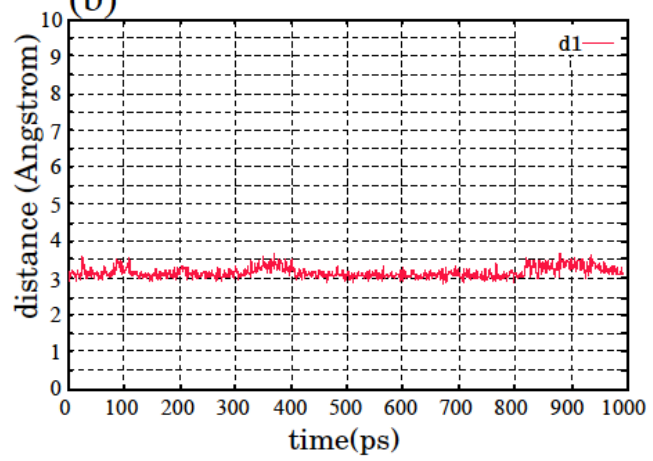

(c)

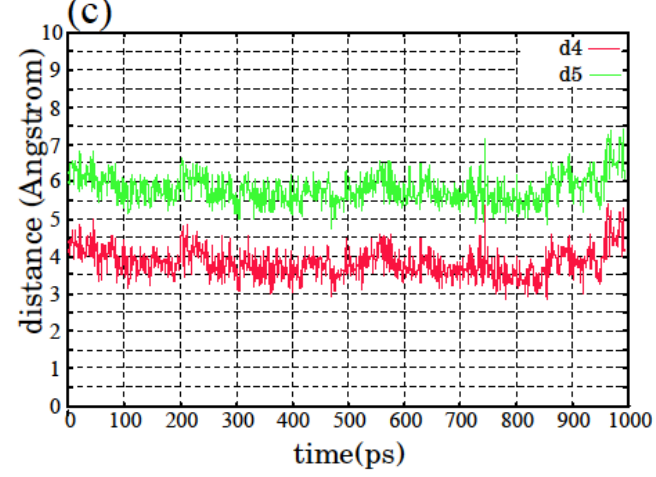

(e)

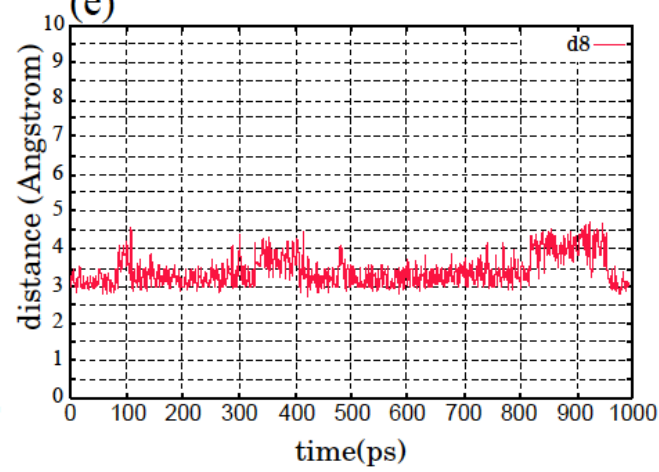

Figure 3

\section{T. Hoshino et al. Behavior of water molecules in ATPase pocket of myosin}


(a)
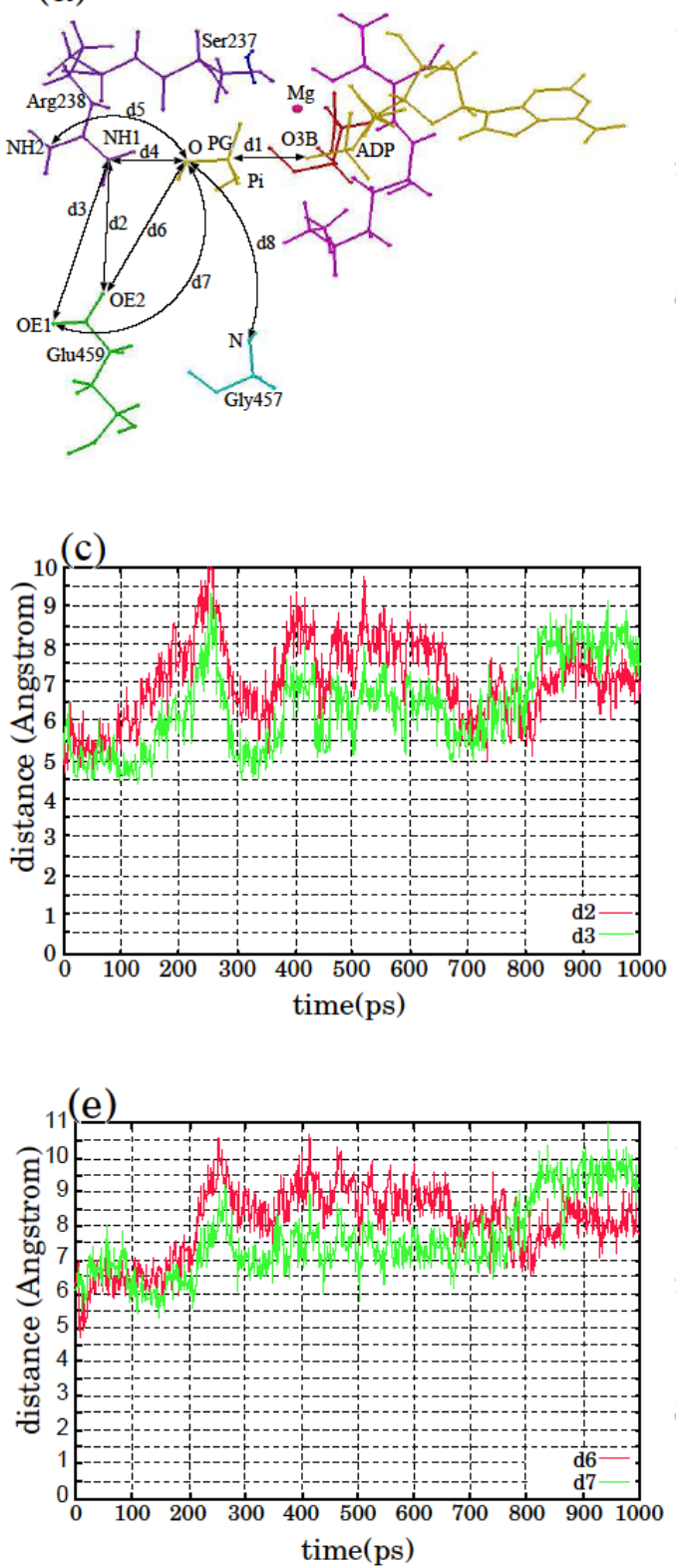

(b)

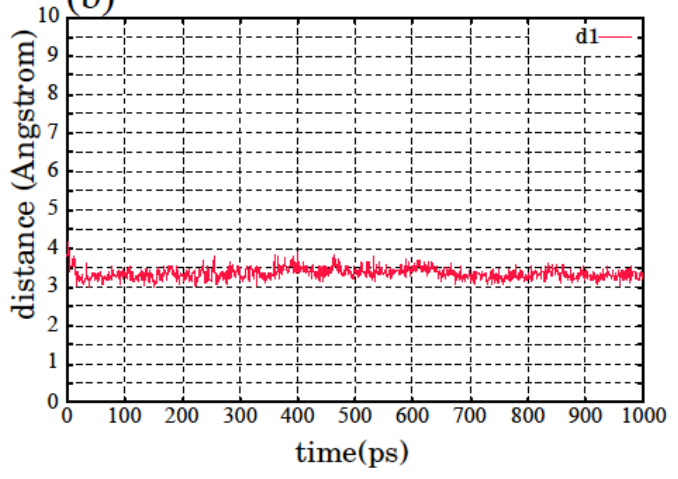

(d)

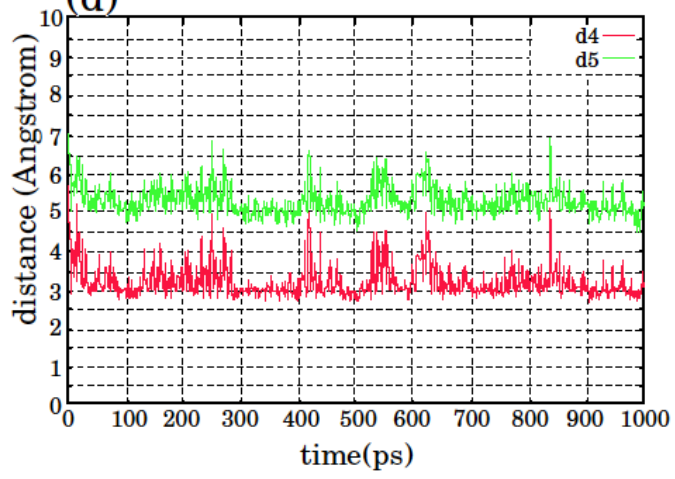

(f)

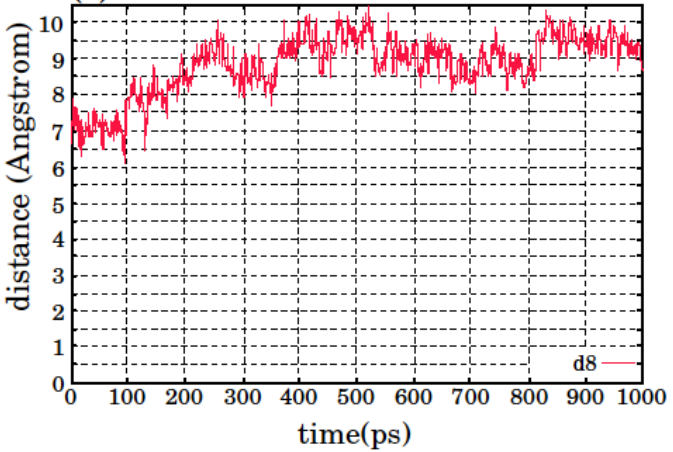

Figure 4

\section{T. Hoshino et al. Behavior of water molecules in ATPase pocket of myosin}


(a)

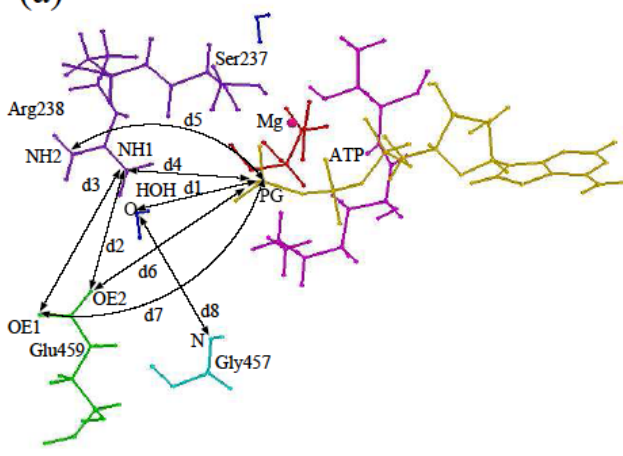

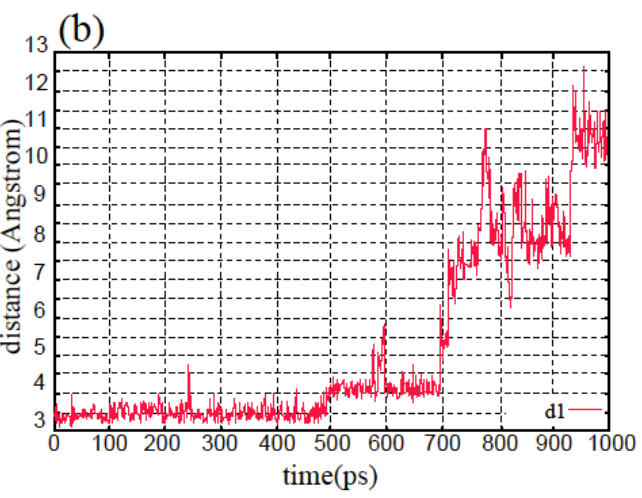

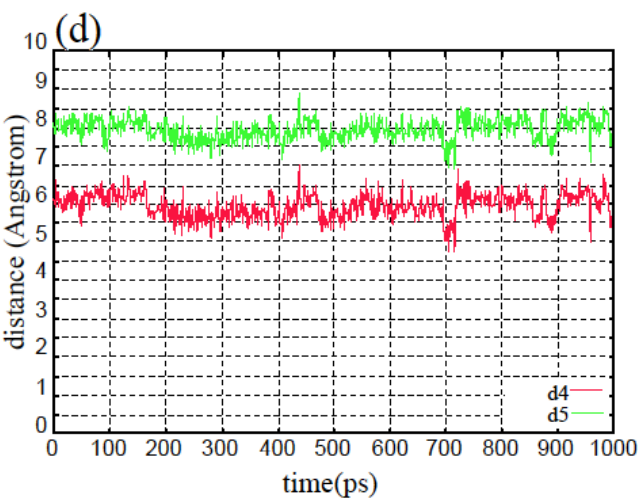

(f)

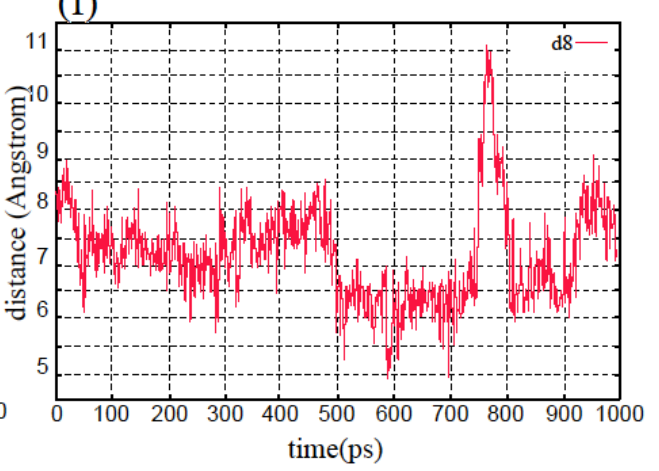

Figure 5

\section{T. Hoshino et al. Behavior of water molecules in ATPase pocket of myosin}


(a)

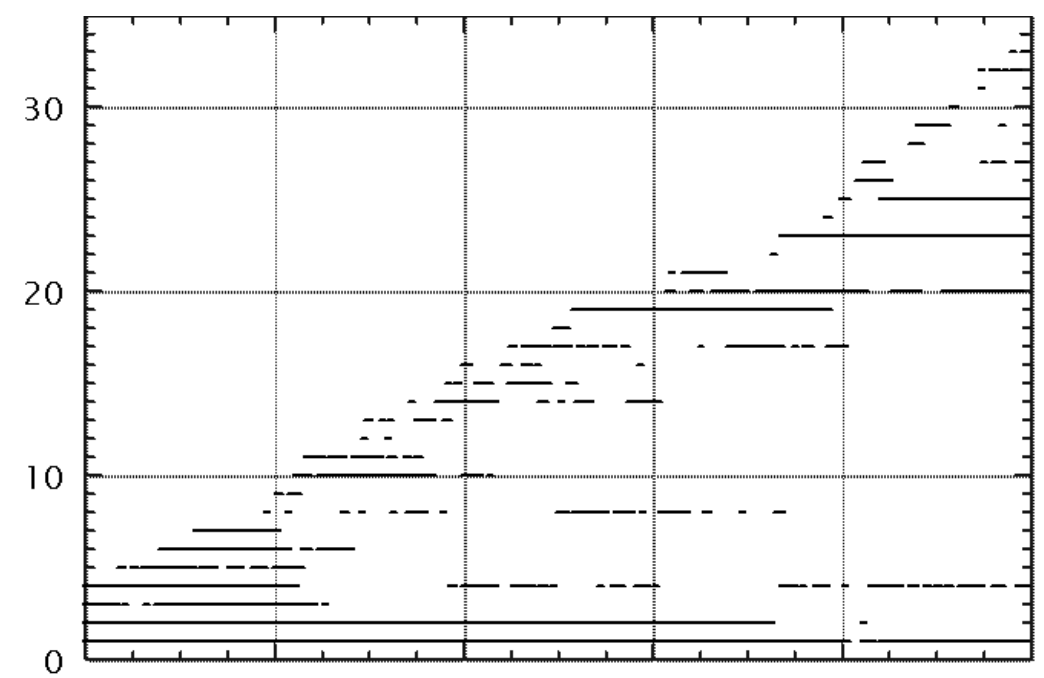

(b)

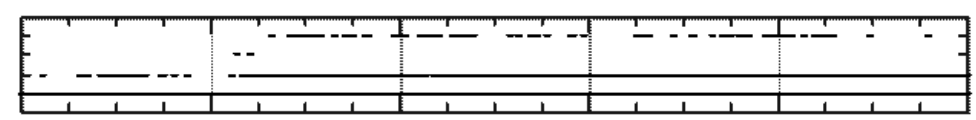

(c)

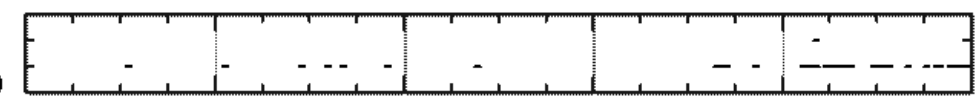

(d)

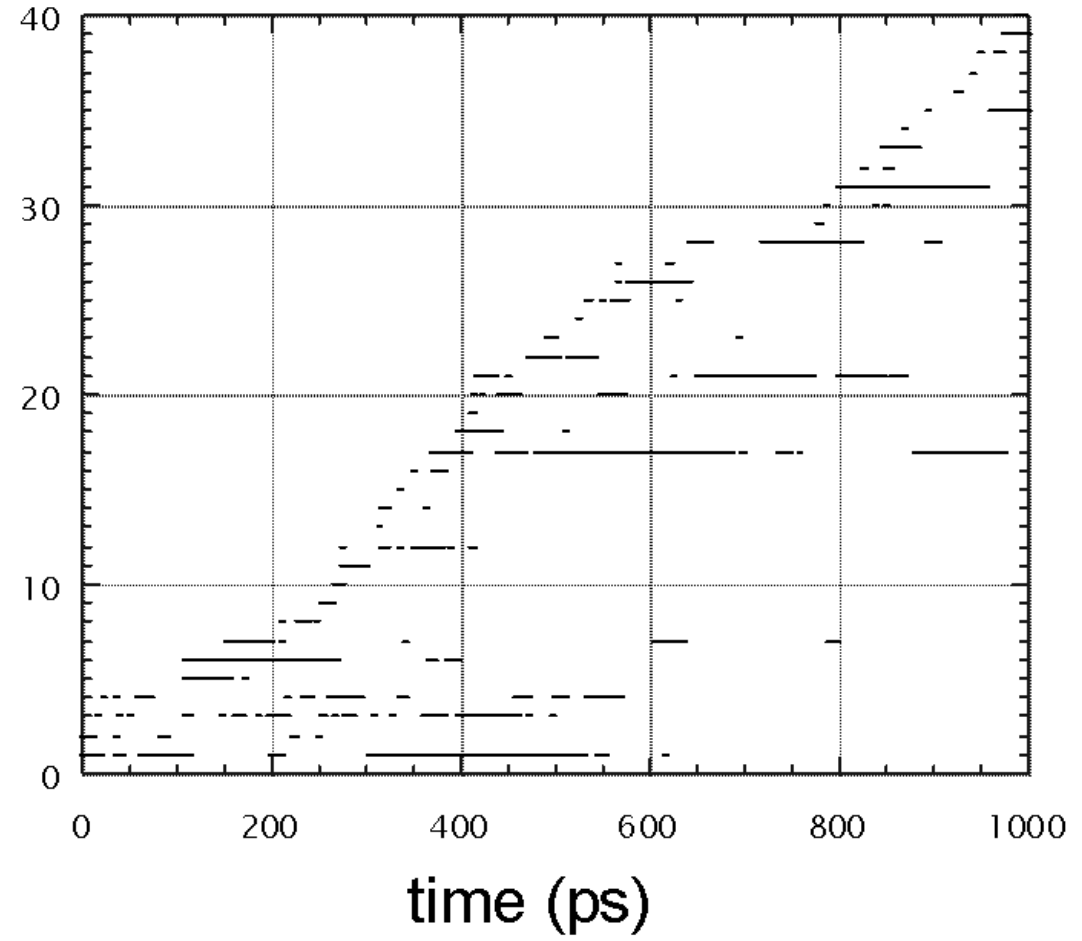

Figure 6 
(a)

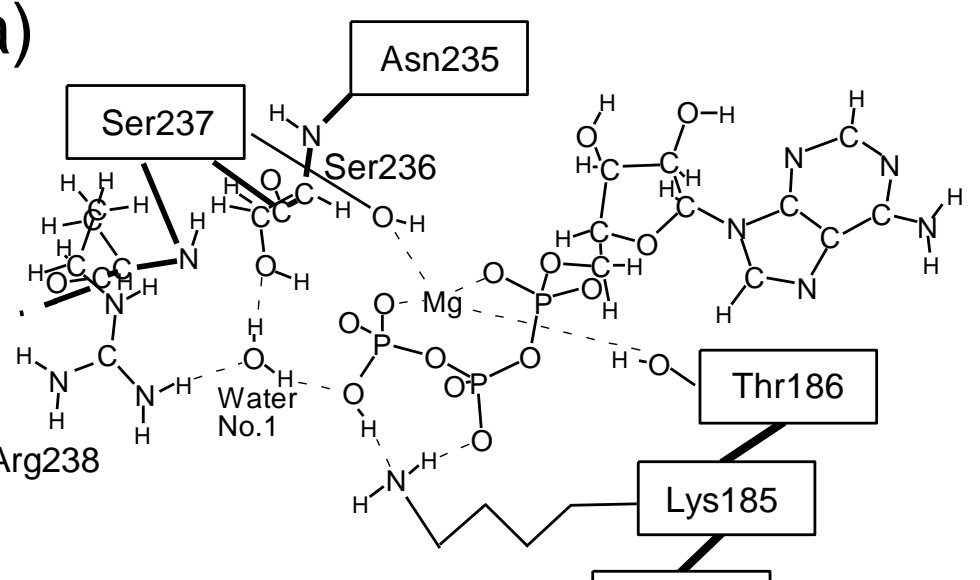

Gly184

Ala183

Glu459

Phe458 Gly457

(b)

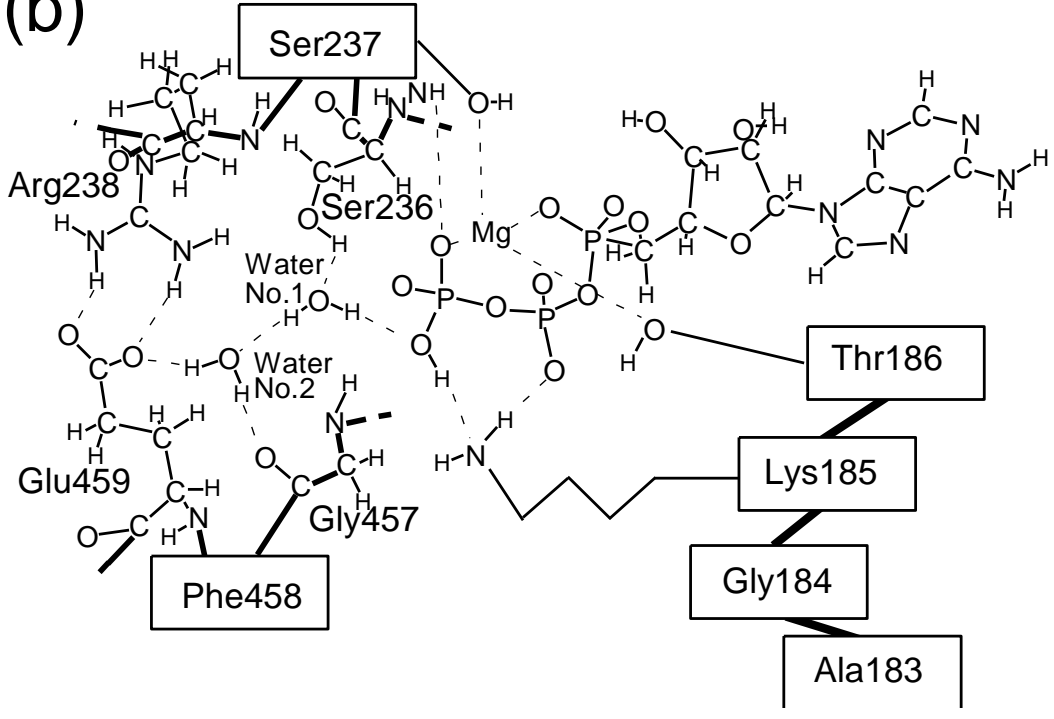

Figure 7

T. Hoshino et al. Behavior of water molecules in ATPase pocket of myosin 